\title{
Acylcarnitine Profiles of Preterm Infants Over the First Four Weeks of Life
}

\author{
JOCHEN MEYBURG, ANDREAS SCHULZE, DIRK KOHLMUELLER, JOHANNES PÖSCHL, \\ OTWIN LINDERKAMP, GEORG F. HOFFMANN, AND ERTAN MAYATEPEK \\ Department of Neonatology [J.M., J.P., O.L.] and Department of General Pediatrics [A.S., D.K., G.F.H., \\ E.M.], Division of Metabolic and Endocrine Diseases, University Children'sHospital, Im Neuenheimer \\ Feld 150, 69120 Heidelberg, Germany
}

\begin{abstract}
ABSTR
Measurement of free carnitine and acylcarnitines allows the
detection of several inborn errors of metabolism in neonatal
screening. Because available data for premature infants is lim-
ited, we studied longitudinal changes in acylcarnitine profiles of
full-term and preterm neonates over the first 4 weeks of life. One
hundred twenty infants were divided into four groups of 30 : A,
gestational age 22 to 27 wk; B, 28 to 31 wk; C, 32 to 36 wk; and
D, 37 to 41 wk. Blood samples spotted on a Guthrie card were
taken on days 5 and 28 . Additional specimens (groups A and B
only) were collected on days 1,3 , 7 , and 14 . Carnitine and its
acyl esters were detected by looking for the precursor ions of m/z
$=85$ using a PE Sciex API 365 electrospray ionization tandem
mass spectrometer. Concentrations of free carnitine and most
acylcarnitines were significantly higher in group A compared
with group D postnatally. Groups B and C displayed intermediate
\end{abstract}
Determination of acylcarnitines by means of tandem mass spectrometry (MS/MS) is of increasing importance in the screening for disorders of inborn errors of metabolism (1-3). Metabolic screening is also applied to preterm infants, although normal ranges are not yet established. However, carnitine levels in cord blood measured by MS/MS differed between term and preterm infants (4). Another study reported increased postnatal plasma and $\mathrm{RBC}$ carnitine levels in preterm infants (5). No sufficient data about dried blood spot acylcarnitine profiles in premature neonates are available so far. Especially in very immature preterm infants, initiation of enteral feeding is often delayed, and total or partial parenteral nutrition is needed for a considerable period of time. Several studies have shown that carnitine levels decrease with total parenteral nutrition in term (6) as well as in preterm infants (7). Therefore, screening for inborn errors of metabolism of preterm infants, e.g. in Germany, is repeated after $2-4 \mathrm{wk}$, when oral feeding

November 14, 2001; April 22, 2002.

Correspondence: Jochen Meyburg, Department of Neonatology, University Children's Hospital, Im Neuenheimer Feld 150, 69120 Heidelberg, Germany; e-mail: jochen_meyburg@med.uni-heidelberg.de

DOI: 10.1203/01.PDR.0000032157.26265.0C values. Carnitine levels in infants from group A and B decreased steadily from day 1 to day 7 , and recovered up to day 14 in group B only. On day 28 carnitine concentrations had further decreased in group A, while reaching postnatal levels again in group B. Postnatal carnitine levels are higher in very immature preterm infants compared with full-term infants, but become lower on day 28. However, the commonly used metabolite ratios should still allow the detection of inborn errors of metabolism. (Pediatr Res 52: 720-723, 2002)
Abbreviations
MS/MS, tandem mass spectrometry
FC, free carnitine
TC, total carnitine
tAC, total acylcarnitine

is well established (8). In this study, we investigated carnitine levels longitudinally in premature neonates by means of MS/ MS, including very immature infants (gestational age from 22 wk) during the first 4 weeks of postnatal life to test the need of individual normal ranges for metabolic screening.

\section{METHODS}

Solvents, reagents and internal standards. High-purity grade methanol was obtained from Merck (Darmstadt, Germany). Butanolic $\mathrm{HCl}(3 \mathrm{M})$ was prepared from high purity butanol (Merck) and HCl-gas. Stable isotopes used as internal standards were obtained from Neo Gen Screening, Pittsburgh, PA, U.S.A.

Patients and blood sampling. We studied a total of 120 neonates, 68 girls and 52 boys, who were divided into four groups of 30 infants each according to their gestational age: A, 22-27 wk/birthweight $780 \pm 170 \mathrm{~g}$ (mean $\pm \mathrm{SD}$ ); B, 28-31 $\mathrm{wk} / 1310 \pm 310 \mathrm{~g} ; \mathrm{C}, 32-36 \mathrm{wk} / 1910 \pm 140 \mathrm{~g}$; and D, 37-41 $w k / 3440 \pm 350$ g. Another 30 healthy term neonates that required a blood sample for other reasons on day 28 (group E), were matched to group D infants, and a dried blood specimen was obtained from excess blood. Infants with severe malfor- 
mations, metabolic diseases, perinatal asphyxia, or proven bacterial infection were excluded from the study. Blood samples were taken from all infants on the 5th postnatal day during routine neonatal screening. Two drops of blood were spotted on a Guthrie card. Additional dried blood specimens of the same infants were obtained in groups A and B on days 1, 3, 7, 14 (excess blood from blood gas samples or electrolyte measurements) and in groups A and B on day 28 (repeated neonatal screening). Oral feeding was started 4 hours after birth. Preterm infants received either expressed breast milk or a preterm milk formula (Pre Beba, Nestlé, Verey, Switzerland). Enteral feedings were increased daily as tolerated. Additional parenteral nutrition not containing carnitine was carried out for a mean of 20 (group A), 16 (group B) and $7 \mathrm{~d}$, respectively (group C). Informed consent had been obtained from all parents before the study. The study was approved by the Ethical Committee of the University Children's Hospital.

Mass spectrometry. Tandem mass spectrometry was carried out as previously described in detail $(1,2,4)$. Briefly, one 3 mm-diameter dot per sample was punched out from a 10 $\mathrm{mm}$-diameter dried blood spot into a vial of a 96-well microtiter-plate. The spots were eluted for $20 \mathrm{~min}$ in $100 \mu \mathrm{L}$ of a methanol stock solution containing internal standards. They were evaporated to dryness in a freeze dryer and then $60 \mu \mathrm{L}$ of $3 \mathrm{~N} \mathrm{HCl}$ in butanol were added. The microtiter-plates were sealed and incubated at $65^{\circ} \mathrm{C}$ in a forced air oven for $15 \mathrm{~min}$. After removal of the seal excess HCl-butanol was evaporated to dryness in a freeze dryer. The derived samples were reconstituted with $100 \mu \mathrm{L}$ acetonitril/water (1:1, vol/vol), containing $0.025 \%$ formic acid. A PE Sciex API 365 triple quadrupole tandem mass spectrometer (PE Sciex, Ontario, Canada) with an ion spray source was used for analysis. Acylcarnitines were detected by looking for the precursor ions of $\mathrm{m} / \mathrm{z}=85$. To allow comparisons with reported values, acylcarnitine concentrations were summarized to obtain levels for short-chain, medium-chain, long-chain and total acylcarnitines as described before (4).

Statistical analysis. The Sudent's $t$ test for unpaired samples was used to test for differences between the individual groups. For the investigation of longitudinal changes in group A, we used the $t$ test for paired samples. Differences were regarded statistically significant at a $p$-level $<0.05$.

\section{RESULTS}

The levels of free carnitine and acylcarnitines on day 5 are summarized in Table 1. Free carnitine and most acylcarnitines were significantly higher in the very immature infants (group A) than in full-term infants (group D). Concentrations of carnitines in the more mature preterm infants (groups B and C) were still higher than in group $\mathrm{D}$, but the differences were less pronounced and only free carnitine as well as a few individual acylcarnitines reached significance (four acylcarnitines in group B and six in group C).

The combined values of total carnitine, total acylcarnitine, medium- and long-chain acylcarnitines on day 5 were significantly higher in the very immature infants of group A when compared with full-term neonates (Fig. 1). The levels in groups $\mathrm{B}$ and $\mathrm{C}$ were intermediate: lower than in group $\mathrm{A}$ and higher although not statistically different from in group D.

In the small preterm infants (groups A and B), carnitine levels were determined consecutively on days 1, 3, 5, 7 and 14

Table 1. Concentrations of free carnitine and acylcarnitines on day 5

\begin{tabular}{|c|c|c|c|c|}
\hline $\begin{array}{c}\text { Group } \\
\text { Gestational age (wk) }\end{array}$ & $\begin{array}{c}\mathrm{A} \\
22-27\end{array}$ & $\begin{array}{c}\text { B } \\
28-31\end{array}$ & $\begin{array}{c}\mathrm{C} \\
32-36\end{array}$ & $\begin{array}{c}\mathrm{D} \\
37-41\end{array}$ \\
\hline Free carnitine & $41 \pm 17 * *$ & $34 \pm 12 *$ & $37 \pm 15^{* *}$ & $28 \pm 10$ \\
\hline \multicolumn{5}{|l|}{ Short-chain acylcarnitines } \\
\hline Acetylcarnitine (C2) & $11.7 \pm 5.4$ & $10.4 \pm 4.2$ & $10.8 \pm 3.4$ & $10.9 \pm 3.6$ \\
\hline Propionylcarnitine (C3) & $2.7 \pm 1.8 * *$ & $1.8 \pm 0.7$ & $1.8 \pm 0.6$ & $1.5 \pm 0.6$ \\
\hline Butyrylcarnitine (C4) & $0.97 \pm 0.65 * *$ & $0.45 \pm 0.17$ & $0.51 \pm 0.12 * *$ & $0.40 \pm 0.12$ \\
\hline Isovalerylcarnitine (C5) & $0.57 \pm 0.40 * *$ & $0.34 \pm 0.12 * *$ & $0.35 \pm 0.12 * *$ & $0.24 \pm 0.06$ \\
\hline \multicolumn{5}{|l|}{ Medium-chain acylcarnitines } \\
\hline Hexanoylcarnitine (C6) & $0.27 \pm 0.17 * *$ & $0.25 \pm 0.13 * *$ & $0.30 \pm 0.17 * *$ & $0.15 \pm 0.10$ \\
\hline Octanoylcarnitine (C8) & $0.23 \pm 0.14 * *$ & $0.17 \pm 0.06$ & $0.22 \pm 0.13 * *$ & $0.15 \pm 0.05$ \\
\hline Octenoylcarnitine (C8:1) & $0.19 \pm 0.14$ & $0.19 \pm 0.13$ & $0.22 \pm 0.14$ & $0.21 \pm 0.07$ \\
\hline Decanoylcarnitine (C10) & $0.17 \pm 0.14^{*}$ & $0.11 \pm 0.05$ & $0.14 \pm 0.06$ & $0.10 \pm 0.03$ \\
\hline Decenoylcarnitine (C10:1) & $0.20 \pm 0.23 *$ & $0.11 \pm 0.05$ & $0.14 \pm 0.06^{*}$ & $0.11 \pm 0.05$ \\
\hline Lauroylcarnitine (C12) & $0.44 \pm 0.55^{*}$ & $0.16 \pm 0.06$ & $0.21 \pm 0.12$ & $0.22 \pm 0.10$ \\
\hline \multicolumn{5}{|l|}{ Long-chain acylcarnitines } \\
\hline Myristoylcarnitine (C14) & $0.38 \pm 0.13^{* *}$ & $0.33 \pm 0.18^{*}$ & $0.30 \pm 0.11$ & $0.25 \pm 0.13$ \\
\hline Myristoleylcarnitine (C14:1) & $0.29 \pm 0.41^{*}$ & $0.17 \pm 0.06$ & $0.18 \pm 0.07$ & $0.14 \pm 0.07$ \\
\hline Palmitoylcarnitine (C16) & $2.69 \pm 0.68^{* *}$ & $2.65 \pm 1.15$ & $2.70 \pm 0.88$ & $2.95 \pm 1.23$ \\
\hline Palmitoyleylcarnitine (C16:1) & $0.30 \pm 0.12 * *$ & $0.28 \pm 0.14 * *$ & $0.22 \pm 0.10$ & $0.20 \pm 0.08$ \\
\hline Stearoylcarnitine (C18) & $1.73 \pm 0.49$ & $1.57 \pm 0.64$ & $1.57 \pm 0.39$ & $1.51 \pm 0.64$ \\
\hline Oleylcarnitine (C18:1) & $2.10 \pm 0.66^{*}$ & $1.70 \pm 0.87$ & $2.05 \pm 0.55$ & $1.84 \pm 0.58$ \\
\hline Hydroxymyristoylcarnitine $(\mathrm{C} 14 \mathrm{OH})$ & $0.10 \pm 0.07$ & $0.09 \pm 0.05$ & $0.15 \pm 0.05$ & $0.06 \pm 0.07$ \\
\hline Hydroxypalmitoylcarnitine $(\mathrm{C} 16 \mathrm{OH})$ & $0.50 \pm 1.07^{*}$ & $0.16 \pm 0.10$ & $0.18 \pm 0.07$ & $0.17 \pm 0.08$ \\
\hline Hydroxypalmitoleylcarnitine $(\mathrm{C} 16: 1 \mathrm{OH})$ & $0.17 \pm 0.11$ & $0.14 \pm 0.08$ & $0.17 \pm 0.07$ & $0.11 \pm 0.08$ \\
\hline Hydroxyoleylcarnitine (C18:10H) & $0.11 \pm 0.08 * *$ & $0.08 \pm 0.05$ & $0.12 \pm 0.07 * *$ & $0.07 \pm 0.04$ \\
\hline
\end{tabular}

Results are expressed as mean $\pm \mathrm{SD}$; unit is $\mu \mathrm{mol} / \mathrm{L}$ for all measurements.

$* p<0.05 / * * p<0.01$ compared to group D. 


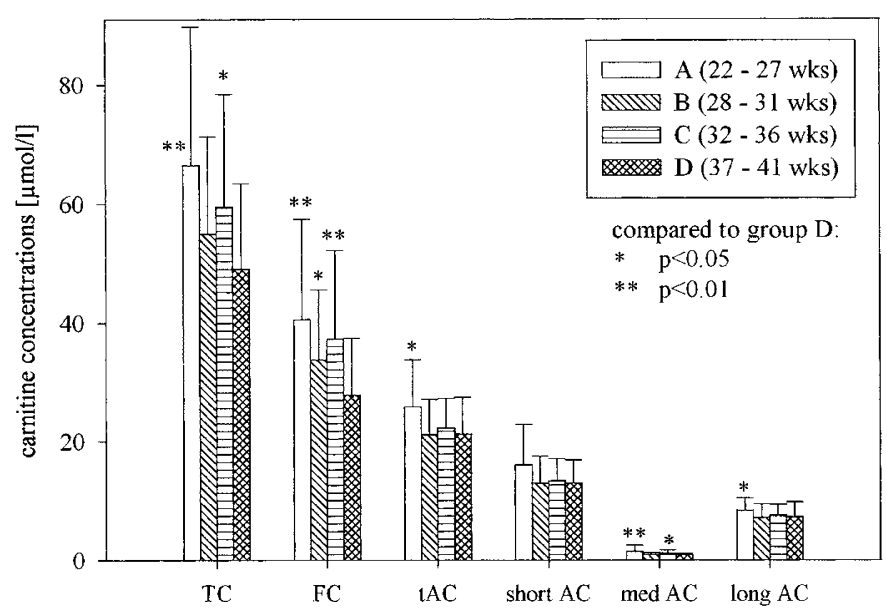

Figure 1. Concentrations of total carnitine (TC), free carnitine (FC), total acylcarnitine (tAC), short-chain acylcarnitines (short AC), medium-chain acylcarnitines (med AC) and long-chain acylcarnitines (long AC) on the fifth postnatal day. Bars and error bars represent mean and SD of the summarized concentrations.

after birth (Fig. 2). In group A, free carnitine, total carnitine, and total acylcarnitine continuously decreased over the whole observation period. In group B, total carnitine, free carnitine, and total acylcarnitine increased postnatally, decreased to day 7 and recovered at days 14 (total and free carnitine) and 28, respectively (total acylcarnitine). After 4 weeks, the very immature infants (group A) showed a $50 \%$ reduction of all carnitines, while the more mature preterm infants (group B) reached their postnatal values again. Carnitine levels in fullterm neonates (group D compared with group E) were about one third higher on day 28 compared with day 5 (data not shown).

\section{DISCUSSION}

The use of new techniques like MS/MS in neonatal screening also requires exact normal values for different subgroups of neonates. Premature infants represent an especially heterogeneous subgroup. Gestational ages may vary from 22 to $36 \mathrm{wk}$, and individually delayed enteral feedings require a repeated metabolic screening after 2-4 wk. Normal values for free carnitine and acylcarnitines in premature infants published so far are restricted either to measurements within the first postnatal days $(5,7)$, small numbers of patients $(9,10)$, measurements at different postnatal ages (11-14), or gestational age above 28 wk $(15,16)$.

The reported levels of total carnitine $(13-31 \mu \mathrm{mol} / \mathrm{L})$, free carnitine $(10-24 \mu \mathrm{mol} / \mathrm{L})$, and total acylcarnitine (3-10 $\mu \mathrm{mol} / \mathrm{L})$ in preterm infants have been obtained using different methods that may yield varying results $(5,7,9-17)$. Some methods underestimate total acylcarnitine and total carnitine due to the loss of long-chain acylcarnitines by extraction (18). In only one study total carnitine was measured simultaneously in term and preterm infants ( 22 versus $29 \mu \mathrm{mol} / \mathrm{L}$ ), and higher levels were found in the latter (5). We also found higher postnatal levels of free carnitine and most acylcarnitines in preterm infants when compared with full-term neonates. More
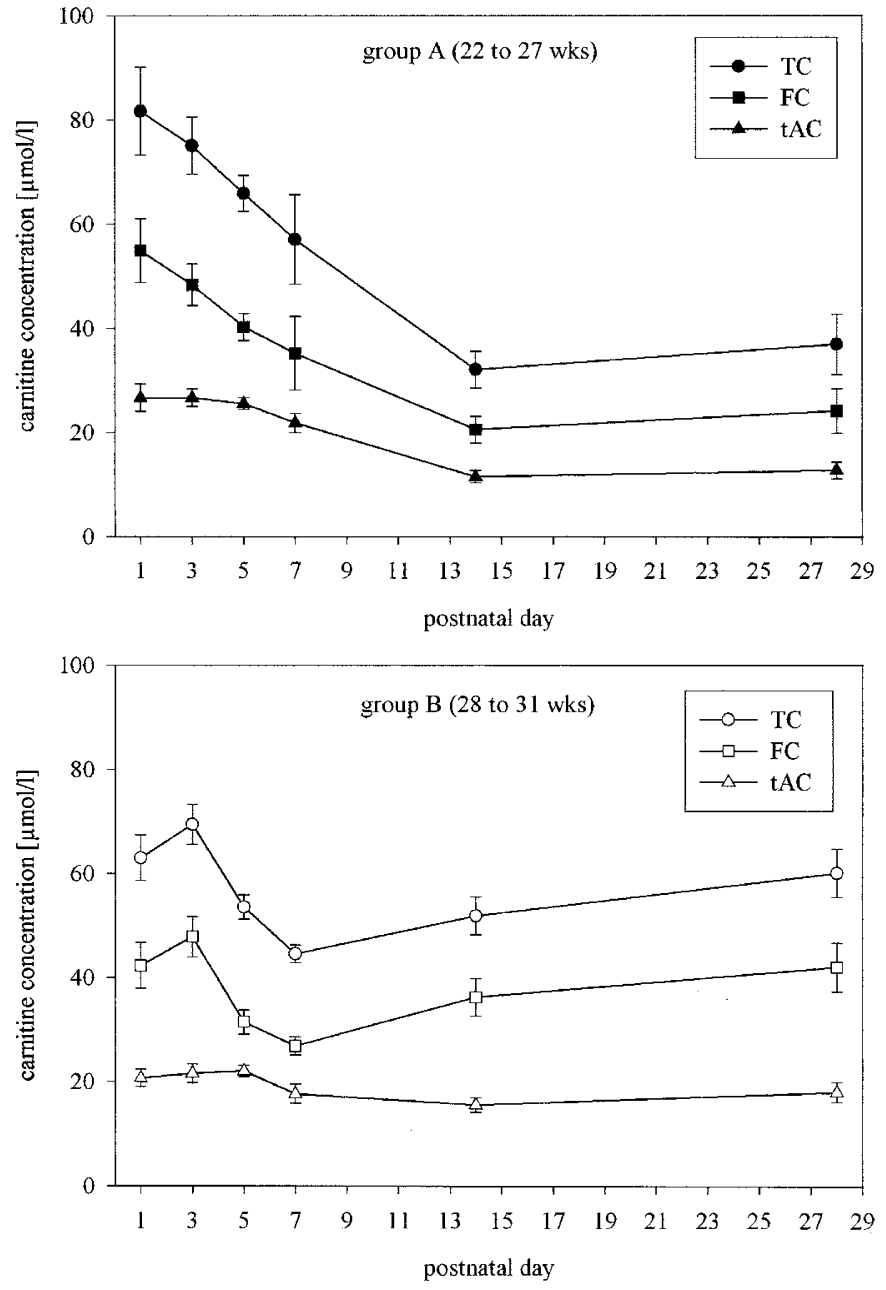

Figure 2. Concentrations of total carnitine (TC), free carnitine (FC) and total acylcarnitine (tAC) during the first 4 weeks of life in group A (upper panel) and group B (lower panel). Values are expressed as mean and SE of mean.

importantly, the differences were markedly higher in very immature children with a gestational age below 28 wk.

It is unlikely that increased postnatal carnitine levels in preterm infants are a result of higher endogenous carnitine production. Decreasing tissue carnitine contents in preterm infants on parenteral nutrition indicate a dependence on exogeneous carnitine supply (19-21). Studies on fetal and maternal plasma carnitine levels suggest a major role of transplacental transfer for the fetal carnitine supply $(22,23)$. The carnitine transportation capacity of the in vitro perfused placenta exceeds the carnitine accumulation of the fetus by far (24). Therefore, fetal carnitine synthesis might be theoretically not required. Fetal plasma carnitine levels obtained by cordocentesis decrease during the course of pregnancy (25) as it was also found for cord blood plasma carnitine levels (5). These observations could well explain our postnatal findings.

In full-term neonates, total carnitine concentrations rise within the first postnatal days, mainly due to an increase of acylcarnitine levels $(4,22)$. A further increase of carnitine concentrations on day 28 has been reported (26). Longitudinal studies on preterm infants are rare and inconsistent. Some studies report a decrease in carnitine levels during the first $2(7$, 
9) and 3 weeks, respectively (10), while all concentrations increased in another (15). The latter finding is unexpected in preterm neonates, since tissue carnitine levels constantly decrease in these infants during the first 4 weeks whereas they rise in full-term neonates (19-21). In the present study, different patterns of postnatal adaptation could be found for different gestational age groups. In contrast to the increasing concentrations of term infants, carnitine levels initially decreased in the relatively mature group B of premature children (27-31 wk), but recovered at 2 weeks of age. This recovery was absent in the very immature group A infants.

\section{CONCLUSIONS}

In conclusion, very immature preterm infants represent a distinct subgroup of neonates in regard to metabolic screening. Compared with full-term infants, markedly higher carnitine concentrations can be found in the first week. A 50\% drop in carnitine levels at the time of a second neonatal screening at 4 weeks of age should be considered normal, although not necessarily physiologic. However, regarding the magnitude of the differences in acylcarnitine concentrations, the commonly used metabolite ratios should still allow the detection of inborn errors of metabolism.

\section{REFERENCES}

1. Chace DH, Hillman SL, van Hove JL, Naylor EW 1997 Rapid diagnosis of MCAD deficiency: quantitatively analysis of octanoylcarnitine and other acylcarnitines in newborn blood spots by tandem mass spectrometry. Clin Chem 43:2106-2113

2. Rashed MS, Ozand PT, Bucknall MP, Little D 1995 Diagnosis of inborn errors of metabolism from blood spots by acylcarnitines and amino acids profiling using automated electrospray tandem mass spectrometry. Pediatr Res 38:324-331

3. Schulze A, Kohlmüller D, Mayatepek E 1999 Sensitivity of electrospray-tandem mass spectro-metry using the phenylalanine/tyrosine-ratio for differential diagnosis of hyperphenylalaninemia in neonates. Clin Chim Acta 283:15-20

4. Meyburg J, Schulze A, Kohlmüller D, Linderkamp O, Mayatepek E 2001 Postnatal changes in neonatal acylcarnitine profile. Pediatr Res 49:125-129

5. Shenai JP, Borum PR, Mohan P, Donlevy SC 1983 Carnitine status at birth of newborn infants of varying gestation. Pediatr Res 17:579-582

6. Schiff D, Chan G, Seccombe D, Hahn P 1979 Plasma carnitine levels during intravenous feeding of the neonate. J Pediatr 95:1043-1046
7. Bonner CM, DeBrie KL, Hug G, Landrigan E, Taylor BJ 1995 Effects of parenteral L-carnitine supplementation on fat metabolism and nutrition in premature neonates. J Pediatr 126:287-292

8. Arbeitsgemeinschaft für Pädiatrische Stoffwechselstörungen (APS) der Deutschen Gesellschaft für Kinderheilkunde 1997 Richtlinien zur Organisation und Durchführung des Neugeborenen-Screenings auf angeborene Stoffwechselstörungen und Endokrinopathien in Deutschland. Der Frauenarzt 38:1180-1184

9. Cederblad G, Svenningsen N 1986 Plasma carnitine and breast milk carnitine intake in premature infants. J Pediatr Gastroenterol Nutr 5:616-621

10. Mares-Perlman JA, Farrell PM, Gutcher GR 1986 Changes in erythrocyte and plasma carnitine concentrations in preterm neonates. Am J Clin Nutr 43:77-84

11. Melegh B, Szücs L, Kerner J, Sandor A 1988 Changes of plasma free amino acids and renal clearances of carnitines in premature infants during L-carnitine-supplemented human milk feeding. J Pediatr Gastroenterol Nutr 7:424-429

12. Melegh B, Kerner J, Sandor A, Vinceller M, Kispal G 1987 Effects of oral L-carnitine supplementation in low-birth-weight premature infants maintained on human milk. Biol Neonate 51:185-193

13. Schmidt-Sommerfeld E, Penn D, Wolf H 1982 Carnitine blood concentrations and fat utilization in parenterally alimented premature newborn infants. J Pediatr 100:260 264

14. Yeh YY, Cooke RJ, Zee P 1985 Impairment of lipid emulsion metabolism associated with carnitine insufficiency in premature infants. J Pediatr Gastroenterol Nutr 4:795798

15. Shortland GJ, Walter JH, Stroud C, Fleming PJ, Speidel BD, Marlow N 1998 Randomised controlled trial of L-carnitine as a nutritional supplement in preterm infants. Arch Dis Child Fetal Neonatal Ed 78:F185-F188

16. Schmidt-Sommerfeld E, Penn D, Wolf H 1983 Carnitine deficiency in premature infants receiving total parenteral nutrition: effect of L-carnitine supplementation. J Pediatr 102:931-935

17. Penn D, Schmidt-Sommerfeld E, Wolf H 1980 Carnitine deficiency in premature infants receiving total parenteral nutrition. Early Hum Dev 4:23-34

18. Fishlock RC, Bieber LL, Snoswell AM 1984 Sources of error in determinations of carnitine and acylcarnitine in plasma. Clin Chem 30:316-318

19. Shenai JP, Borum PR 1984 Tissue carnitine reserves of newborn infants. Pediatr Res 18:679-682

20. Penn D, Ludwigs B, Schmidt SE, Pascu F 1985 Effect of nutrition on tissue carnitine concentrations in infants of different gestational ages. Biol Neonate 47:130-135

21. Penn D, Schmidt-Sommerfeld E, Pascu F 1981 Decreased tissue carnitine concentrations in newborn infants receiving total parenteral nutrition. J Pediatr 98:976-978

22. Novak M, Monkus EF, Chung D, Buch M 1981 Carnitine in the perinatal metabolism of lipids. I. Relationship between maternal and fetal plasma levels of carnitine and acylcarnitines. Pediatrics 67:95-100

23. Cederblad G, Niklasson A, Rydgren B, Albertsson-Wikland K, Olegard R 1985 Carnitine in maternal and neonatal plasma. Acta Paediatr Scand 74:500-504

24. Schmidt-Sommerfeld E, Penn D, Sodha RJ, Prögler M, Novak M, Schneider H 1985 Transfer and metabolism of carnitine and carnitine esters in the in vitro perfused human placenta. Pediatr Res 19:700-706

25. Bernardini I, Evans MI, Nicolaides KH, Economides DL, Gahl WA 1991 The fetal concentrating index as a gestational age-independent measure of placental dysfunction in intrauterine growth retardation. Am J Obstet Gynecol 164:1481-1487

26. Campoy C, Bayes R, Peinado JM, Rivero M, Lopez C, Molina-Font JA 1998 Evaluation of carnitine nutritional status in fullterm newborn infants. Early Human Dev 53(Suppl):S149-S164 